\title{
The Effect of Oral Pregabalin on Epidural-Induced Shivering and Epidural Characteristics in Gynecological Surgeries, a Prospective Randomized Double-Blind Study
}

\section{Tarek Al Menesy, MD ( $\square$ tarek_gada@yahoo.com )}

Anesthesia department, Faculty of medicine, Beni Suef University https://orcid.org/0000-0002-91116660

Ghada Adel, MD

Anesthesia department, Faculty of medicine, Cairo University

Ahmed A. Badawy, MD

Anesthesia department, Faculty of medicine, Cairo University

Samaa A. Kasem, MD

Anesthesia department, Faculty of medicine, Beni Suef University

Ashraf Abd Elmawgoud, MD

Anesthesia department, Faculty of medicine, Cairo University

Yasmin A. Badawy

Faculty of Medicine, Cairo University

\section{Research Article}

Keywords: Pregabalin, Shivering, Epidural shot, Onset and Duration

Posted Date: December 12th, 2018

DOI: https://doi.org/10.21203/rs.2.76/v1

License: (a) (i) This work is licensed under a Creative Commons Attribution 4.0 International License.

Read Full License 


\section{Abstract}

Purpose: The previous reports tried to reduce shivering and improve neuraxial anesthesia characteristics by the systemic use of different drugs. This study was directed to evaluate the effect of pregabalin premedication on both shivering and epidural characteristics following single shot loading.

Patients and Methods: Eighty female patients, ASA grade I and II, scheduled for gynecological surgeries under epidural anesthesia were studied. The patients were divided into two groups: Pregabalin $(P)$ group and Control (C) group in which the patients received $150 \mathrm{mg}$ of pregabalin and placebo capsules respectively sixty minutes prior to surgery. Following epidural loading, the onset and degree of shivering were compared between the two groups. Also, the epidural onset, level and duration were compared. The perioperative hemodynamics, sedation scores, meperidine consumption and side effects were followed up and registered.

Results: The onset of shivering was significantly prolonged and the degree of shivering was less in pregabalin group compared to Control group. The duration of epidural shot was shorter in the control group in relation to the pregabalin treated patients. Regarding both onset and level of block, no significant changes were detected. The patients were more sedated with less meperidine consumption in the pregabalin group. The two groups were similar as regards the perioperative hemodynamics and occurrence of side effect.

Conclusion: The premedication with pregabalin reduced onset and degree of shivering and prolonged the duration of single shot epidural block.

\section{Introduction}

Neuroaxial anesthesia carries a high incidence of shivering similar to that occurring following general anesthesia. The sympathetic block and consequently both vasodilatation and redistribution of heat from central to peripheral areas were believed to be the main etiology of that shivering. In addition to the unpleasant sensation and dissatisfied response of patients to the anesthetic practice, shivering is documented to increase oxygen consumption, vasoconstriction which may increases the risk of critical post operative complications like myocardial infarction as well as both metabolic and hormonal changes [1-3].

Due to the hazardous effects of shivering, several studies tried to control shivering by both pharmacologic [2] and non- pharmacologic interventions [4, 5].

Pharmacologically, opioids, alpha-2 agonists [6-9], ketamine [10] physostigmine [11], nefopam, tramadol [12], granisetron [13], doxapram, ketanserine [2] and dexamethasone [14] succeeded to reduce shivering. Also, amino acid infusion exerted a positive feedback in that field [15]. 
Pregabalin with its gabapentinoid structure was included in management of both convulsive and anxiety disorders in addition to the control of neuropathic pain. It binds to the voltage-dependent calcium channel in the central nervous system and consequently decreases the release of the excitatory neurotransmitters including glutamate, norepinephrine, substance $\mathrm{P}$ and calcitonin gene-related peptide. Through its central action, pregabalin was believed to reduce neuronal excitability $[16,17]$. Previous reports included pregabalin in the control of postoperative pain following both general and regional anesthesia. The total opioid consumption and pain scores were reduced significantly when traced postoperatively [17-20]. Even pregabalin succeeded to reduce the post dural puncture headache following spinal anesthesia [21].

When tested in general anesthesia, Ozgencil et al [18] found that pregabalin had a significant effect in minimizing shivering in the postoperative period. Although neuraxial anesthesia was believed to exert high incidence of shivering [1], but to our knowledge the previous studies did not include pregabalin in the control of shivering following epidural anesthesia.

Therefore, this study was directed to evaluate the effect of pregabalin $150 \mathrm{mg}$ oral premedication in reducing the perioperative shivering which may accompany epidural anesthesia, and may also improve the quality of epidural block and consequently affect the onset, level and duration of single shot epidural anesthesia.

\section{Patients And Methods}

After obtaining the ethical committee approval of Faculty of medicine, Beni Suef University (FM-BSU) in Egypt and an informed consent from the patients. Eighty female patients (30-40 years old) with an American society of Anesthegiologists physical status (ASA) I-II, scheduled for gynecological surgery (Posterior repair and transobturator transfixation) under epidural anesthesia, were enrolled in this randomized prospective double -blind study from May to November 2018. The present study was taken place in Beni Suef university hospital (where all patients recruited) and was registered at ClinicalTrials.gov with Identification number: NCT03721432 and registration date: October 24, 2018.

The patients were excluded from the study if ASA class was more than II, anticipated difficult epidural insertion, any contraindication to epidural anesthesia, if any patient developed wet or bloody tab, or the patients were under psychotropic or antiepileptic therapy.

The 80 patients were randomly allocated into one of two groups (using sealed opaque numbered envelopes indicating the group of each patient, carried out by an independent anesthesiologist and given to the ward nurse) [40 patients in each group]:

- Pregabalin group $(\mathrm{P}),[n=40]$. received pregabalin $150 \mathrm{mg}$ capsules (Lyrica $\Theta$, Pfizer) sixty minutes prior to the epidural insertion.

Control group (C), [n=40]: received placebo capsules sixty minutes prior to epidural insertion. 
Prior to administration of study drugs, mean arterial blood pressure (MAP), heart rate (HR), oxygen saturation (SpO2), and tympanic temperature ( $\mathrm{T}$ temp) were taken as [baseline reading]. No sedative premedication was allowed. Upon reaching the operation theater, a second set of readings were taken [preoperative reading], together with sedation score using a 4 points "Modified Wilson" Sedation Scale [22] [1=awake; 2=drowsy but arousable to command; $3=$ asleep but arousable to mild physical stimulation; and 4= asleep but not arousable to mild physical stimulation].

On the operating table and after attaching the standard monitoring [5 lead ECG, pulse oximetry, noninvasive arterial blood pressure], the epidural anesthesia was standardized for all patients. After intravenous infusion of $500 \mathrm{ml}$ of lactated Ringer's solution in a wide-bore cannula, the epidural catheter was inserted in a lateral position. Following the exclusion of both subarachnoid and intravascular insertion of the catheter, a loading epidural dose of $10 \mathrm{ml} \mathrm{levo-bupivacaine} 0.5 \%$ and $50 \mathrm{mcg}$ fentanyl were injected.

The operating room temperature was adjusted at $22 \circ \mathrm{C}$ and all patients were actively warmed using a convection machine (hot air blower) with warming of all I.V fluids.

The hemodynamic readings [MAP, HR and SpO2] were monitored and recorded every 5 minutes (as a routine practice) and tympanic temperature [T temp] was taken upon arrival to operating room, after activation of epidural, then every 15 minutes till the end of surgery.

Intraoperatively, arterial blood pressure was maintained within $20 \%$ of the baseline readings using intravenous boluses of Lactated Ringer's solution and $6 \mathrm{mg}$ increments of ephedrine (if required). Bradycardia (HR less than 60 beats per minutes) was treated with intravenous atropine boluses $0.6 \mathrm{mg}$.

Shivering (as a primary outcome) was graded by a blinded observer during the intraoperative and postoperative period using the scale validated by Crossley and Mahajan [23] and Tsai and Chu [24]: [0= no shivering, $\mathbf{1}=$ piloerection or peripheral vasoconstriction but no visible shivering, $\mathbf{2}=$ muscular activity in only one muscle group, $3=$ muscular activity in more than one muscle group but not generalized shivering, 4= shivering involving the whole body]. Grades 3, and 4 shivering for at least 3 minutes were considered positive, and maximum shivering was considered if generalized shivering interfering with ECG monitoring. Positive shivering or lower grade shivering but described as distressful by the patient were treated with intravenous administration of meperidine $20 \mathrm{mg}$. If no improvement after this management, the patient was excluded from the study as higher doses of meperidine and small dose of ketamine were used to manage that sever shivering.

For the selection of safe and effective anti-shivering treatment, the medications were revised and meperidine in this small dose was picked up due to its effectiveness in treating shivering without significant changes in the epidural when compared to ketanserine, doxapram, clonidine, magnesium, ketamine, orphenadrine, methylphenidate, pentazocin and nefopam $[2,25]$. 
At the end of surgery, the patients were transferred to PACU (for one hour), where hemodynamics and tympanic temperature were recorded (every 15 minutes). Shivering was also observed and scaled and treated the same way as intraoperatively. Patients were only discharged from PACU if shivering score were $\leq 2$ and modified Aldrete score was $\geq 9$ [26].

Postoperatively, when the patients started to experience pain at the surgical site and according to the acute pain management protocol by the anesthesia department in the hospital, a loading of $8 \mathrm{ml}$ levobupivacaine $0.125 \%$ was given followed by infusion of levo-bupivacaine $0.125 \%$ and fentanyl $2 \mathrm{mcg} / \mathrm{ml}$ at a rate of $6 \mathrm{ml} / \mathrm{hour}$.

Any perioperative complications (e.g. bradycardia, hypotension, de-saturation or nausea and vomiting) were observed, registered and treated appropriately.

The following were observed (by anaesthetist blinded to the studied drugs):

Demographic data (age, weight and height), duration of surgery (in minutes).

- Tympanic temperature ( $T$ temp.) in Celsius degree as baseline (before drug intake), preoperative (on arrival to operating room), after epidural loading, then every 15 minutes intraoperative.

- Shivering (as a primary outcome): incidence of positive shivering (at any time), incidence of maximum shivering (at any time) and median and range of shivering severity between the two studied groups.

Sedation: assessed 60 minutes after studied drug in take (preoperatively).

- Epidural characteristics (as secondary outcome): onset of the block (the time from epidural loading until feeling of numbness in the lower extremities), duration of the block (the time from the onset till starting feeling of pain at the surgical site) and level of the block (assessed by the ice technique).

- Meperidine consumption: average consumed dose of meperidine in both groups.

\section{Statistical analysis:}

Sample size was calculated after a pilot study where the difference in incidence of shivering between groups was $30 \%$. By using the power analysis $80 \%$ at level of significance 0.05 [ $\beta=0.8$ and $\alpha=0.05$ ], the minimum number of patients in each group was 34 , therefore, the number increased to 40 patients to compensate any dropout.

Statistical analysis was performed using PASW Statistics 18 (SPSS Inc., Chicago, IL, USA). Data were reported as [mean \pm SD], [median (range)] or number and percentage. Parametric data were analysed using an unpaired Student's t-test. Ordinal data were analysed using the Mann-Whitney ranked sum test. Nominal data were analysed using either the chi-square or Fisher's exact test.

\section{Results}


The results of the present study showed no statistically significant difference between the two studied group as regards the demographic data of the patients (age, weight and height), and the operative duration. But sedation score, measured upon reaching the operating room (OT), 60 minutes after studied

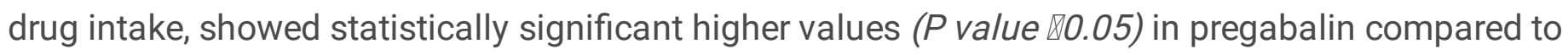
placebo premedicated patients [Table 1].

Although, the incidence of positive shivering was statistically significant lower in pregabalin group compared to the controlled group [14/39 (35\%) and 23/38(60\%), respectively] but the incidence of maximum shivering with median and range of its severity did not show any statistically significant difference between the two studied groups. The significant difference in the incidence of positive shivering was reflected on the statistically significant lower average consumed dose of meperidine used to treat the shivering in pregabalin group compared to the controlled group $[7.1 \pm 9.7$ and $11.7 \pm 9.9 \mathrm{mg}$, respectively], [Table 2].

As regards the epidural block characteristics, both the onset and level of block showed no statistically significant difference between the two studied groups. Although the duration of epidural block was statistically prolonged in pregabalin group compared to the control group, yet, it was not clinically significant being [106 \pm 12.9 and $93 \pm 7.4$ ] minutes, respectively, [Table 3].

The tympanic temperature in the two studied groups showed no statistically significant difference all through the reading intervals perioperatively, [Figure 2].

No complications recorded among the patients in either group.

\section{Discussion}

The present study showed that pregabalin statistically reduced the incidence of occurrence of positive shivering but not the maximum or average intensity and also statistically extended the duration of epidural block which was not clinically significant.

When tested in general anesthesia, Ozgencil et al., [18] found that pregabalin had a significant effect in minimizing shivering in the postoperative period. Although neuraxial anesthesia was believed to exert high incidence of shivering with incidence up to $30 \%$ due to vasodilatation and transfer of heat from central to peripheral zones in response to sympathetic block $[1,2]$ but, to our knowledge, the previous studies did not include pregabalin in the control of shivering following epidural injection.

The present study was designed to examine the efficacy of preemptive pregabalin in controlling shivering which occurs after epidural anesthesia loading. The researchers were inspired with the following factors: 1- In a previous study [18] and as a secondary outcome, gabapentinoids; gabapentin and pregabalin successfully reduced the postoperative shivering after general anesthesia, 2-Pregabalin showed improvement in the management of postdural puncture headache [21], 3-The detrimental effect of shivering on the susceptible cardiopulmonary patients as it increases the cardiac output, heart rate and 
oxygen consumption up to $600 \%$ [3], 4- The associated tremors may affect the accuracy of monitor devices and alter the patient satisfaction [3], 5- The previous studies up to date, did not include pregabalin in the control of shivering following epidural anesthesia.

The result of the present study was going in hand with the observational outcome in the study of Ozgencil et al., [18] who found that pregabalin $150 \mathrm{mg}$ twice daily and gabapentin $600 \mathrm{mg}$ twice daily after lumbar laminectomy and discectomy surgery under general anesthesia, succeeded to reduce postoperative shivering when compared to placebo group.

Previous studies examined the efficacy of multiple drugs in the management of postoperative shivering and showed controversial results.

In accordance with the present study, intravenous nefopam $0.15 \mathrm{mg} / \mathrm{kg}$ compared to $0.5 \mathrm{mg} / \mathrm{kg}$ intravenous tramadol showed a promising effect in the control shivering after mepivacaine $1 \mathrm{mg} / \mathrm{kg}$ epidurally or $0.2 \mathrm{mg} / \mathrm{kg}$ in subarachnoid block [12]. Controversially, when compared to nefopam, Röhm et al [11] reported that physostigmine superiorly reduced both incidence and severity of post general anesthetic shivering.

Also, adding pethidine $10 \mathrm{mg}$ to intrathecal bupivacaine in the study of Hong et al., [7] provided superior reduction effect of both shivering incidence and severity when compared to morphine $0.1 \mathrm{mg}$ or $0.2 \mathrm{mg}$.

Coinciding with the present study, Kose et al., [10] demonstrated an effective control of shivering when intravenous ketamine $0.25 \mathrm{mg} / \mathrm{kg}$ and $0.5 \mathrm{mg} / \mathrm{kg}$ were given prior to intrathecal injection of bupivacaine. Also, the intraoperative loading with dexmedetomidine $1 \mathrm{mcg} / \mathrm{kg}$ for $10 \mathrm{~min}$ followed by an infusion of $0.4 \mathrm{mcg} / \mathrm{kg} / \mathrm{hr}$ reduced the incidence of shivering significantly so that only 7 patients shivered when compared to 21 patients in placebo treated group [27].

Following general anesthesia, granisetron in the study of Iqbal et al., [13] showed an effective prophylactic anti-shivering profile similar to the outcome of pethidine $25 \mathrm{mg}$.

Contradictory to these results, ondansetron $8 \mathrm{mg}$ administered before combined spinal epidural anesthesia failed to show significant reduction in the incidence and severity of postoperative shivering in the study of Browning et al., [28].

When introduced to anesthesia field, pregabalin was proved not only to control postoperative pain but also to exert an effective perioperative sedative action. The present study showed statistically significant sedation 60 minutes after pregabalin intake compared to the placebo treated group.

Similar to the result of the present study, White et al., [29] found that, pregabalin in a dose dependent manner demonstrated a higher sedation scores up to 120 minutes in PACU when compared to $75 \mathrm{mg}$ and $150 \mathrm{mg}$. Also, Gonano et al., [30] reported that preemptive pregabalin exerted a significant anxiolytic effect in 40 patients underwent minor orthopedic procedures. 
But Tahereh Eskandarian et al., [31], showed that pregabalin in children underwent dental procedure reduced the VAS-sedation score starting from 2 hours post-dose and reached the maximum within 2-4 hours with behavioral rating showing no statistically significant difference from the placebo. They concluded that, pregabalin anxiolytic and sedative effects in children can be anticipated 2 hours after pregabalin intake.

The majority of previous studies traced the effect of the epidural injection of different adjuvant drugs on the block characteristics but only few reports studied the systemic use of these drugs on the epidural analgesic outcome.

The systemic intravenous administration of dexmedetomidine in the study of Elvan et al., [27] showed a longer duration time of block following epidural injection when compared to intravenous saline use.

Also and similar to that, the oral clonidine 90 minutes prior to epidural morphine $2 \mathrm{mg}$ in the study of Goyagi et al., [9] improved postoperative analgesia and reduced intratravenous PCA morphine requirements up to 42 hours following abdominal hysterectomy surgeries. Also, the study of Suzuki et al., [32], the intravenous administration of low dose ketamine $0.05 \mathrm{mg} / \mathrm{kg} / \mathrm{hr}$ following thoracotomy augmented the analgesic effect of epidural morphine and ropivacaine with reduction of pain scores up to 48 hours postoperatively.

During labour epidural analgesia, the pregabalin $150 \mathrm{mg} / 12$ hours reduced the total consumption of ropivacaine and sufentanil in the patient controlled epidural analgesia (PCEA) set protocol. Also, through modulation of visceral sensitization, the top up requests during delivery was significantly decreased when compared to the prazepam $10 \mathrm{mg}$ every 12 hours [33].

\section{Limitations of the present study:}

The main limitation of the present study was the insufficient literature about pregabalin use with neuraxial blockade to examine its effect on shivering and block characteristics so that the researchers were cautious not to use a dose-dependent fashion to test different doses of pregabalin or multiple doses on a daily basis perioperatively.

\section{Conclusion}

Oral intake of pregabalin $150 \mathrm{mg} / \mathrm{kg}$ (60 minutes prior to epidural anesthesia) reduced the incidence of shivering and seemed to prolong the duration of single shot epidural dose in gynaecological surgery.

\section{Declarations}

Ethics approval and Consent to participate: the study protocol was approved by the ethical committee of Faculty of medicine, Beni Suef University (FM-BSU), Egypt and informed consent was obtained to participate this study from the patients. 
Availability of data and materials: The datasets generated during and/or analysed during the current study are not publicly available due to confidentiality of FM-BSU files but are available from the corresponding author on reasonable request.

Competing interests: The authors declare that they have no competing interests.

Funding: the current study was funded by FM-BSU as regards the drug, equipments and data.

Authors' contributions: T.M and S.K. put the design and the protocol and shared in the clinical part, G.A. and A.A.B shared in writing the manuscript and carried out the statistical data analysis, A.M shared in writing the manuscript and revising the data analysis and Y.A.B. shared in collecting the background resources and writing the manuscript. All authors read and approved the final manuscript.

\section{References}

1. Hoshijima H, Takeuchi R, Kuratani N, Nishizawa S, Denawa Y, Shiga T, et al. Incidence of postoperative shivering comparing remifentanil with other opioids: a meta-analysis. J Clin Anesth. 2016;32:300-312.

2. Peter Kranke, Leopold H. Eberhart, Norbert Roewer, and Martin R. Tramer. Pharmacological Treatment of Postoperative Shivering: A Quantitative Systematic Review of Randomized Controlled Trials. Anesth Analg 2002;94:453-60.

3. Dal D, Kose A, Honca M, Akinci SB, Basgul E, Aypar U. Efficacy of prophylactic ketamine in preventing postoperative shivering. Br J Anaesth. 2005;95(2):189-92.

4. Najafianaraki A, Mirzaei K, Akbari Z, Macaire P. The effects of warm and cold intrathecal bupivacaine on shivering during delivery under spinal anesthesia. Saudi J Anaesth. 2012,6(4):336-40.

5. Horn EP, Bein B, Böhm R, Steinfath M, Sahili N, Höcker J. The effect of short time periods of preoperative warming in the prevention of peri-operative hypothermia. Anaesthesia. 2012;67(6):612-7.

6. Bicer C, Esmaoglu A, Akin A, Boyaci A. Dexmedetomidine and meperidine prevent postanaesthetic shivering. Eur J Anaesthesiol. 2006;23(2):149-53.

7. Hong JY, Lee IH. Comparison of the effects of intrathecal morphine and pethidine on shivering after Caesarean delivery under combined-spinal epidural anaesthesia. Anaesthesia. 2005;60(12):1168-72.

8. Akin A, Ocalan S, Esmaoglu A, Boyaci A. The effects of caudal or intravenous clonidine on postoperative analgesia produced by caudal levobupivacaine in children. Paediatr Anaesth. 2010;20(4):350-5.

9. Goyagi T, Tanaka M, Nishikawa T. Oral clonidine premedication enhances postoperative analgesia by epidural morphine. Anesth Analg. 1999;89(6):1487-91. 
10. Kose EA, Honca M, Dal D, Akinci SB, Aypar U. Prophylactic ketamine to prevent shivering in parturients undergoing Cesarean delivery during spinal anesthesia. J Clin Anesth. 2013;25(4):275-80.

11. Röhm KD, Riechmann J, Boldt J, Schuler S, Suttner SW, Piper SN. Physostigmine for the prevention of postanaesthetic shivering following general anaesthesia - a placebo-controlled comparison with nefopam. Anaesthesia. 2005;60(5):433-8.

12. Bilotta F, Pietropaoli P, Sanita' R, Liberatori G, Rosa G. Nefopam and tramadol for the prevention of shivering during neuraxial anesthesia. Reg Anesth Pain Med. 2002;27(4):380-4.

13. Iqbal A, Ahmed A, Rudra A, Wankhede RG, Sengupta S, Das T, Roy D. Prophylactic granisetron vs pethidine for the prevention of postoperative shivering: a randomized control trial. Indian J Anaesth. 2009;53(3):330-4.

14. Entezariasl M, Isazadehfar K. Dexamethasone for prevention of postoperative shivering: a randomized double-blind comparison with pethidine. Int J Prev Med. 2013;4(7):818-24.

15. Inoue S, Shinjo T, Kawaguchi M, Nakajima Y, Furuya H. Amino acid infusions started after development of intraoperative core hypothermia do not affect rewarming but reduce the incidence of postoperative shivering during major abdominal surgery: a randomized trial. J Anesth. 2011;25(6):850-4.

16. Taylor CP, Angelotti T, Fauman E. Pharmacology and mechanism of action of pregabalin: the calcium channel alpha2-delta (alpha2-delta) subunit as a target for antiepileptic drug discovery. Epilepsy Res. 2007;73(2):137-50.

17. Bornemann-Cimenti H, Lederer AJ, Wejbora M, Michaeli K, Kern-Pirsch C, Archan S, Rumpold-Seitlinger G, Zigeuner R, Sandner-Kiesling A. Preoperative pregabalin administration significantly reduces postoperative opioid consumption and mechanical hyperalgesia after transperitoneal nephrectomy. $\mathrm{Br} J$ Anaesth. 2012;108(5):845-9

18. Ozgencil E, Yalcin S, Tuna H, Yorukoglu D, Kecik Y. Perioperative administration of gabapentin 1,200 mg day-1 and pregabalin 300 mg day-1 for pain following lumbar laminectomy and discectomy: a randomised, double-blinded, placebo-controlled study. Singapore Med J. 2011;52(12):883-9.

19. Dauri M, Faria S, Gatti A, Celidonio L, Carpenedo R, Sabato AF. Gabapentin and pregabalin for the acute post-operative pain management. A systematic-narrative review of the recent clinical evidences. Curr Drug Targets. 2009;10(8):716-33.

20. Yücel A, Ozturk E, Aydoğan MS, Durmuş M, Colak C, Ersoy MÖ. Effects of 2 different doses of pregabalin on morphine consumption and pain after abdominal hysterectomy: a randomized, doubleblind clinical trial. Curr Ther Res Clin Exp. 2011;72(4):173-83.

21. Huseyinoglu U, Huseyinoglu N, Hamurtekin E, Aygun H, Sulu B. Effect of pregabalin on post-duralpuncture headache following spinal anesthesia and lumbar puncture. J Clin Neurosci. 2011;18(10):1365- 
22. Höhener D, Blumenthal S. and Borgeat A. Sedation and regional anaesthesia in the adult patient. $\mathrm{Br} J$ Anaesth. 2008;100(1):8-16.

23. Crossley AWA, Mahajan RP. The intensity of postoperative shivering is unrelated to axillary temperature. Anaesthesia 1994;49:205-7.

24. Tsai YC, Chu KS. A comparison of tramadol, amitriptyline, and meperidine for postepidural anesthetic shivering in parturients. Anesth Analg 2001;93:1288-92.

25. Park SM, Mangat HS, Berger K, Rosengart AJ. Efficacy spectrum of antishivering medications: metaanalysis of randomized controlled trials. Crit Care Med. 2012;40(11):3070-82.

26. Thomas WF, Macario A. The postanesthesia care unit. In: Miller RD, editor. Anesthesia. Philadelphia: Churchill Livingstone; 2005. p. 2708-9.

27. Elvan EG, Oç B, Uzun S, Karabulut E, Coşkun F, Aypar U. Dexmedetomidine and postoperative shivering in patients undergoing elective abdominal hysterectomy. Eur J Anaesthesiol. 2008;25(5):357-64.

28. Browning RM, Fellingham WH, O'Loughlin EJ, Brown NA, Paech MJ. Prophylactic ondansetron does not prevent shivering or decrease shivering severity during cesarean delivery under combined spinal epidural anesthesia: a randomized trial. Reg Anesth Pain Med. 2013;38(1):39-43.

29. White PF, Tufanogullari B, Taylor J, Klein K. The effect of pregabalin on preoperative anxiety and sedation levels: a dose-ranging study. Anesth Analg. 2009;108(4):1140-5.

30. Gonano C, Latzke D, Sabeti-Aschraf M, Kettner SC, Chiari A, Gustorff B. The anxiolytic effect of pregabalin in outpatients undergoing minor orthopaedic surgery. J Psychopharmacol. 2011;25(2):249-53.

31. Tahereh Eskandarian, Hamidreza Eftekharian, and Rojin Soleymanzade. Efficacy and safety of premedication with single dose of oral pregabalin in children with dental anxiety: A randomized doubleblind placebo-controlled crossover clinical trial. Dent Res J(Isfahan). 2015;12(6):528-33.

32. Suzuki M1, Haraguti S, Sugimoto K, Kikutani T, Shimada Y, Sakamoto A. Low-dose intravenous ketamine potentiates epidural analgesia after thoracotomy. Anesthesiology. 2006;105(1):111-9.

33. Lavand'homme PM, Roelants F. Evaluation of pregabalin as an adjuvant to patient-controlled epidural analgesia during late termination of pregnancy. Anesthesiology. 2010;113(5):1186-91.

\section{Tables}

Table (1): Demographic data, operative duration and preoperative sedation score of the studied groups. Data represented as [mean \pm SD] or [median (range)]: 


\begin{tabular}{lccc}
\hline & $\begin{array}{c}\text { Group (C) } \\
(n=38)\end{array}$ & $\begin{array}{c}\text { Group (P) } \\
(n=39)\end{array}$ & $\begin{array}{c}P \\
\text { value }\end{array}$ \\
\hline Age (year) & $39.6 \pm 3$ & $38.8 \pm 3.1$ & 0.132 \\
Body weight $(\mathrm{kg})$ & $71.7 \pm 8.7$ & $74.6 \pm 9.1$ & 0.08 \\
Height $(\mathrm{cm})$ & $160.9 \pm 5.2$ & $162.5 \pm 4$ & 0.08 \\
Duration of surgery (minutes) & $61.5 \pm 8$ & $63.9 \pm 9.2$ & 0.09 \\
Preoperative Sedation score & $1(1-2)$ & $2(1-3) *$ & 0.000 \\
\hline
\end{tabular}

Group $(C)=$ control, Group $(P)=$ pregabalin

* Statistically significant compared to group $(C)$, [P value $[0.05]$

Table (2): Incidence of positive, maximum, median of severity of shivering, total and average meperidine consumption. Data represented as number and percentage (\%), [median (range)] or [mean $\pm \mathrm{SD}]$ :

\begin{tabular}{lccc}
\hline & $\begin{array}{c}\text { Group (C) } \\
(n=38)\end{array}$ & $\begin{array}{c}\text { Group (P) } \\
(n=39)\end{array}$ & $\begin{array}{c}P \\
\text { value }\end{array}$ \\
\hline $\begin{array}{l}\text { Incidence of positive shivering at any time [number } \\
(\%)]\end{array}$ & $\begin{array}{c}23 / 38 \\
(60 \%)\end{array}$ & $\begin{array}{c}14 / 39 \\
(35 \%)\end{array}$ & 0.01 \\
Incidence of maximum shivering at any time [number & $9 / 38(23 \%)$ & $8 / 39(20 \%)$ & 0.06 \\
$(\%)]$ & & & \\
$\begin{array}{l}\text { Median and range of severity of shivering } \\
\text { Average meperidine consumption }(\mathrm{mg})\end{array}$ & $3(1-4)$ & $2(1-4)$ & 0.07 \\
\hline
\end{tabular}

Group $(C)=$ control, Group $(P)=$ pregabalin

* Statistically significant compared to group (C), [P value $[0.05]$

Table (3): Epidural Characteristics (Onset, Duration and Level of block). Data represented as $[$ mean $\pm \mathrm{SD}]$ or [median (range)]:

\begin{tabular}{lccc}
\hline & $\begin{array}{c}\text { Group (C) } \\
(n=38)\end{array}$ & $\begin{array}{c}\text { Group (P) } \\
(n=39)\end{array}$ & $\begin{array}{c}P \\
\text { value }\end{array}$ \\
\hline Onset of epidural block (minutes) & $8.7 \pm 1.3$ & $8.2 \pm 1.6$ & 0.056 \\
Duration of epidural block (minutes) & $93 \pm 7.4$ & $106 \pm 12.9 *$ & 0.007 \\
Level of epidural block (segments) & $10(6-10)$ & $8(6-10)$ & 0.195 \\
\hline
\end{tabular}

Group $(C)=$ control, Group $(P)=$ pregabalin

* Statistically significant compared to group (C), [P value $[0.05]$

\section{Figures}




\section{Flow Diagram}

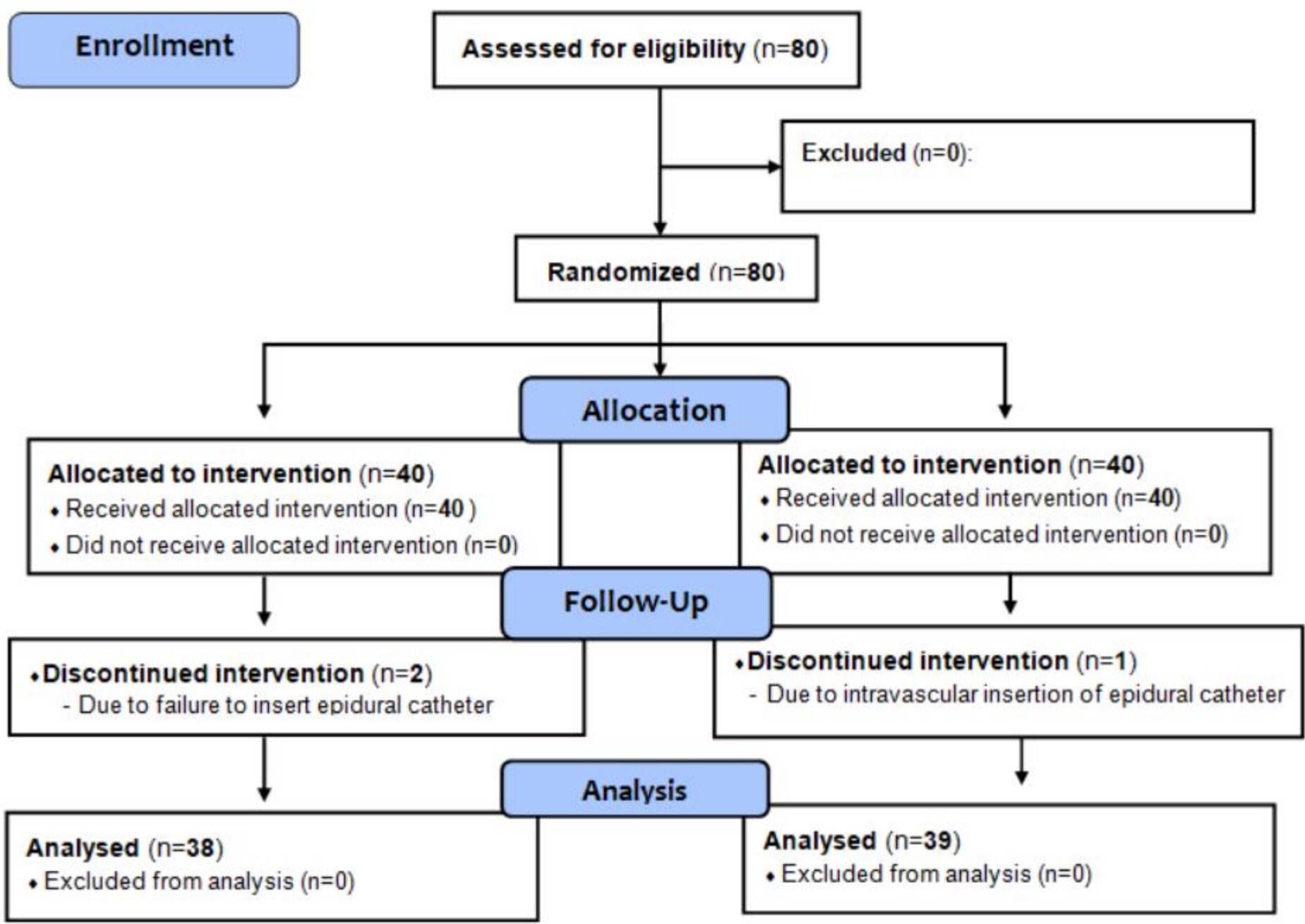

Figure 1

Flow diagram of the studied groups 


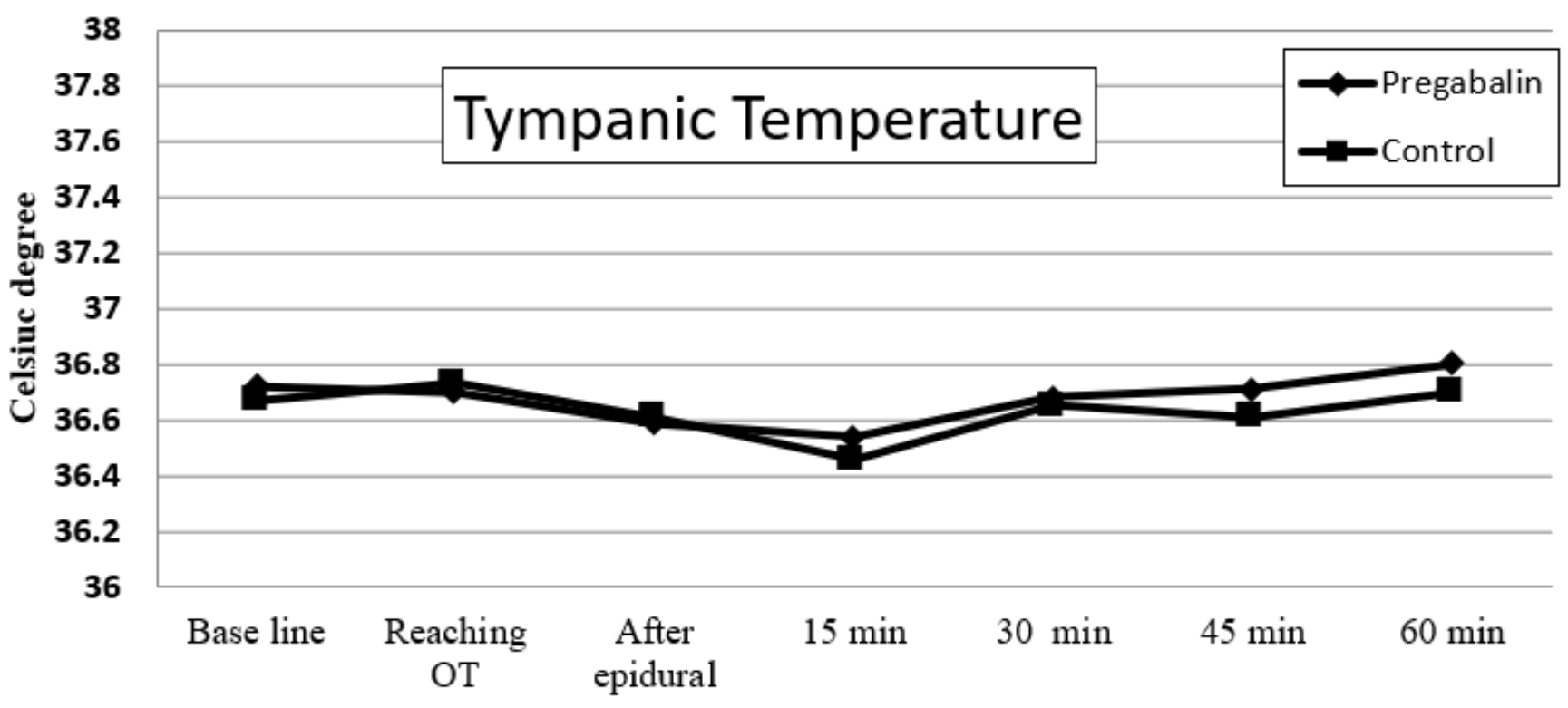

Figure 2

Tympanic Temperature (T Temp.) in Celsius degree 